\title{
Ensuring Informed Decision-Making for Cancer Screening
}

\author{
Stephen H. Bradley, MRCP, Matthew J. Thompson, MPH, DPhil, and \\ Brian D. Nicholson, MRCGP
}

The history of cancer screening has demonstrated that the case for cancer screening is not straightforward. In contemporary practice, sharing decision-making with patients has become expected of family physicians. At the same time, increasing emphasis has been placed on encouraging patients to participate in screening programs to improve cancer outcomes. The success of cancer screening is often judged by the number of those who participate. Improving cancer outcomes should be a priority for family medicine, but the importance of this goal should not undermine doctors' commitment to helping patients make informed decisions that are consistent with their values and priorities. If we are serious about empowering patients, we need to be more open about the limitations of cancer screening, to help patients make up their minds. (J Am Board Fam Med 2021;34:435-438.)

Keywords: Cancer Screening, Clinical Decision-Making, Family Medicine, Family Physicians, Patient Participation, Shared Decision-Making

For much of its history, cancer screening has been presented as a 'no-brainer' with self-evident benefits for those diagnosed 'early'. ${ }^{1}$ We now have a greater understanding that the trade-offs resulting from overdiagnosis and overtreatment are far from straightforward. ${ }^{2,3}$ The precise calculus to quantify these trade-offs varies depending on cancer, screening modality, or schedule. To be justified, any harm must be offset by benefits such as increased longevity and improved quality of life. The rationale for screening not captured by the evidence may well exist, such as the desire of individuals to be reassured by negative tests, or to know if cancer is present. The desire for reassurance for patients and

This article was externally peer reviewed.

Submitted 10 July 2020; revised 26 August 2020; accepted 2 September 2020.

From the Leeds Institute of Health Sciences, University of Leeds, UK (SHB); Department of Family Medicine, University of Washington, Seattle (MJT); Nuffield Department of Primary Care Health Sciences, University of Oxford, UK (BDN).

Funding: Stephen Bradley is a Clinical Research Fellow and Matthew Thompson is an Associate Director of the multi-institutional CanTest Collaborative, which is funded by Cancer Research UK (C8640/A23385). The opinions expressed in this article are those of the authors alone and do not represent the opinions of Cancer Research UK or the authors' employers.

Conflict of interest: The authors have no conflicts of interest to declare. their doctors is entirely natural but unfortunately the absolute reduction in risk of cancer following a negative test is marginal and the 'interval' cancers that develop between screening rounds often have poor outcomes. ${ }^{2,4}$ If there are other credible justifications for screening, we should be explicit about these as well as our desire to 'save lives.'

These debates rage within medical communities but, very few patients seem to be aware of the controversies that surround the screening programs they attend. ${ }^{5-7}$ Diagnosing asymptomatic patients is not unique to cancer screening. Many patients who see their physician for unrelated problems are diagnosed with hypertension following an opportunistic blood pressure check. But cancer screening is different. Testing can be unpleasant for patients and positive screens may lead to further invasive procedures. Cancer represents a heterogeneous spectrum of diseases and it is impossible to know what the natural history of many screen-detected cancers would have been if left undetected. ${ }^{8}$

Given the difficultly in navigating these complex decisions, it would seem reasonable for patients to

Corresponding author: Dr Stephen Bradley, MRCP, Leeds Institute of Health Sciences, Rm 10.36 Worsley Building, Leeds, LS2 9JT, United Kingdom, Phone: +44 (0)113 34 30806 (E-mail: medsbra@leeds.ac.uk). 
be able to rely on the guidance of their physicians. Unfortunately, many doctors mistakenly attribute increased detection as evidence that cancer screening saves lives. ${ }^{9}$ Clinicians cannot know the evidence base for every intervention. Acting within the bounds of established practice and national recommendations form the basis of prudent and defensible practice.

Although bodies such as the National Screening Committee in the United Kingdom (UK) and the United States Preventive Services Task Force (USPSTF) are charged with evaluating the evidence for screening, experience has shown that their recommendations can be side-lined. Examples include the overturning of the recommendation against screening women under 50 years for breast cancer in the United States, and the UK's decision to make prostate cancer screening available if requested. ${ }^{10-12}$ Given the many different pressures that drive cancer screening policy, including political considerations and campaigning from patient groups and physician specialty organizations, this is hardly surprising. ${ }^{13-15}$

In the evaluation of established screening programs, the volume of uptake is prioritized as a measure of success, rather than achieving informed decision making that might jeopardize rates of screening uptake. ${ }^{16-18} \mathrm{We}$ require new metrics that demonstrate patients' participation in decision making to inform reimbursement and evaluation. Instead of simply incentivizing screening uptake, such reforms could remunerate clinicians for exploring the benefits and harms of individual screening programs. Alternatively, auditing or surveying could take place as to the level of participation patients felt that they had in screening decisions. We also need to be prepared to accept that if we embark on genuinely shared decision making, in which we are honest about harms and uncertainties as well as possible benefits, declining screening will be an appropriate choice for some patients.

Screening recommendations are shaped by societal and political pressures and informed by a population health perspective as well as by evidence that individuals who take part may benefit. Patients need independent support to decide for themselves. Decision aids and improved information for patients are necessary first steps. ${ }^{15-17}$ Bodies like the National Institute for Health and Care Excellence (NICE) and the USPSTF could support individual decision making by commissioning objective and up to date and innovative web resources such as infographics, animations, and accessible literature to explain the harms and benefits of the major screening programs in ways that are easily understood. Discussing screening in terms of 'natural frequencies' can help to communicate the scale of benefits and harms. ${ }^{19}$ For example, 1000 person tools such as those produced by the Canadian Task Force on Preventive Health Care and the updated breast cancer screening information produced by the National Health Service in England aim to achieve a more intuitive understanding using diagrams, which represent 1000 individuals, some of whom are shaded in different colors to represent different outcomes. ${ }^{20-22}$ Such tools, and the spirit that inspired them, could be widely emulated although barriers such as lack of physician time may well impede their uptake in the consulting room.

For many patients, it would also be helpful to know how their peers would make decisions on cancer screening when adequately apprised of the evidence and arguments. ${ }^{23-25}$ In recent years citizens' juries have become increasingly used to guide fraught public policy decisions. The process involves selecting a representative jury from the population, who deliberate following detailed evidence sessions which may span several days. They have already been used to shape cancer policy in Australia and New Zealand. ${ }^{23-26}$ The value of such juries is that they bring diverse perspectives on issues, which require reference to society's values, in addition to factual evidence. Such juries seem well placed to inform individual decision making through knowledge as to what a representative sample of their peers recommended, given the opportunity of days to consider the evidence and deliberate on a decision. The purpose of this would not be to deliver the 'right' decision but to give individuals insight into what a sample of their peers would recommend, after exhaustively considering all the complex issues involved in a way that cannot be achieved in the consulting room. Over several decades the methodology of citizens' juries has become well established and experience has demonstrated that for these assemblies to have a useful role it is vital that they are as representative as possible of the population and that each jury's remit and procedures are carefully planned. ${ }^{23,27}$

For all the lip service paid to patient autonomy, failing to acknowledge the complexity of cancer 
screening perpetuates paternalism in medicine. If we are serious about shared decision making, even a goal as worthy as reducing cancer deaths does not override our responsibility to be open about the limitations of cancer screening, lest patients fail to make 'the right' decision. Some patients may prefer to defer to physicians' interpretation of national clinical guidelines rather than grappling with the complexities of these choices. But for those who wish to be completely engaged in health care decisions, we have a responsibility to help them find their preferred balance between the benefits and harms of cancer screening. Providing accessible, objective information is essential. But we should also consider exploring different strategies such as citizen's juries and aligning the metrics of cancer screening with patient autonomy, as well as with uptake.

To see this article online, please go to: http://jabfm.org/content/ 34/2/435.full.

\section{References}

1. American Cancer Society advertisement, "If you haven't had a mammogram you need more than your breasts examine". Available at: https://www. latimes.com/science/sciencenow/la-sci-sn-breast-cancerscreening-mammograms-20161012-snap-story.html. Accessed November 24, 2019.

2. Raffle AE, Mackie A, Gray JAM. Screening Evidence and Practice. Oxford University Press; 2007;215-256.

3. Marmot MG, Altman DG, Cameron DA, Dewar JA, Thompson SG, Wilcox M. The benefits and harms of breast cancer screening: an independent review. Br J Cancer 2013;108:2205-40.

4. Kirsh VA, Chiarelli AM, Edwards SA, et al. Tumor characteristics associated with mammographic detection of breast cancer in the Ontario breast screening program. J Natl Cancer Inst 2011;103: 942-50.

5. Baum M. Harms from breast cancer screening outweigh benefits if death caused by treatment is included. BMJ 2013;346:f385.

6. Prasad V, Lenzer J, Newman DH. Why cancer screening has never been shown to "save lives"and what we can do about it. BMJ 2016;352:h6080.

7. McCartney M. Margaret McCartney: Can we now talk openly about the risks of screening? BMJ 2018;361:k2055.

8. Welch HG. The heterogeneity of cancer. Breast Cancer Res Treat 2018;169:207-8.

9. Wegwarth O, Schwartz LM, Woloshin S, Gaissmaier W, Gigerenzer G. Do Physicians Understand Cancer Screening Statistics? A National Survey of Primary
Care Physicians in the United States. Ann Intern Med 2012;156:340-9.

10. Fletcher SW. Whither Scientific Deliberation in Health Policy Recommendations? - Alice in the Wonderland of Breast-Cancer Screening. N Engl J Med 1997;336:1180-3.

11. National Health Service. PSA testing prostate cancer. 2018. Available at: https://www.nhs.uk/conditions/ prostate-cancer/psa-testing/. Accessed August 25, 2020.

12. Wise J. Mobile lung cancer testing in supermarket car parks is to be expanded. BMJ 2017;359: j5 5450 .

13. Mahase E. Will genome testing of healthy babies save lives? BMJ 2019;367:16449.

14. Fixing the present building for the future: newborn screening for rare conditions. 2019. Available at: http:// www.geneticalliance.org.uk/wp-content/uploads/ 2019/07/FIXING-THE-PRESENT-BUILDINGFOR-THE-FUTURE-Newborn-screening-for-rareconditions-.pdf. Accessed 24 Nov 2019.

15. Detailed ACR Statement on Ill-Advised and Dangerous USPSTF Mammography Recommendations. 2009. Available at: https://www.acr.org/Advocacy-andEconomics/ACR-Position-Statements/Ill-Advisedand-Dangerous-USPSTF-Mammography-Recommendations. Accessed 06 May 2020.

16. McCartney $M$. Cancer screening review diminishes informed choice., BMJ 2019;367:16224.

17. Richards $M$. Report of the independent review of adult screening programmes in England. 2019. Available at: https://www.england.nhs.uk/wp-content/uploads/ 2019/02/report-of-the-independent-review-of-adultscreening-programme-in-england.pdf. Accessed August 25, 2020.

18. Smith SK, Trevena L, Simpson JM, Barratt A, Nutbeam D, McCaffery KJ. A decision aid to support informed choices about bowel cancer screening among adults with low education: randomised controlled trial. BMJ 2010;341:c5370.

19. Spiegelhalter DJ. Understanding uncertainty. Ann Fam Med 2008;6:196-7.

20. Lung cancer-1000 person tool. https://canadiantaskforce. ca/tools-resources/lung-cancer-2/lung-cancer-forpatients/. Accessed November 24, 2019.

21. Scalia P, Durand M-A, Faber M, Kremer JA, Song J, Elwyn G. User-testing an interactive option grid decision aid for prostate cancer screening: lessons to improve usability. BMJ Open 2019;9:e026748.

22. NHS breast screening: helping you decide. Available at: https://assets.publishing.service.gov.uk/government/ uploads/system/uploads/attachment_data/file/840343/ Breast_screening_helping_you_decide.pdf. Accessed August 25, 2020.

23. Rychetnik L, Carter SM, Abelson J, et al. Enhancing citizen engagement in cancer screening 
through deliberative democracy. J Natl Cancer Inst 2013;105:380-6.

24. Mosconi P, Colombo C, Satolli R, Carzaniga C. Involving a citizens' jury in decisions on individual screening for prostate cancer. PLOS ONE 2016;11 (1): $\mathrm{e} 0143176$.

25. Wise J. Citizens' juries for health policy. BMJ 2017;357:j2650.
26. Degeling C, Barratt A, Aranda S, et al. Should women aged $70-74$ be invited to participate in screening mammography? A report on two Australian community juries. BMJ Open 2018;8: $\mathrm{e} 021174$.

27. The Jefferson Centre, citizens juries. https:// jefferson-center.org/about-us/how-we-work/. Accessed November 25, 2019. 